\title{
Biological equivalent dose studies for dose escalation in the stereotactic synchrotron radiation therapy clinical trials
}

\author{
Y. Prezado ${ }^{a)}$ \\ ID17 Biomedical Beamline, European Synchrotron Radiation Facility (ESRF), 6 rue Jules Horowitz, \\ BP 220, 38043 Grenoble Cedex, France \\ G. Fois \\ ID17 Biomedical Beamline, European Synchrotron Radiation Facility (ESRF), 6 rue Jules Horowitz, \\ BP 220, 38043 Grenoble Cedex, France and Dipartimento di Fisica, Universitá degli Studi di Cagliari, \\ S.P. Monserrato Sestu km 0.700, Monserrato, Cagliari 09042, Italy \\ M. Edouard \\ Equipe 6, Institut National de la Santé et de la Recherche Médicale U836, 6 rue Jules Horowitz, BP 220, \\ 38043 Grenoble Cedex, France; Université Joseph Fourier, BP 53, 38041 Grenoble Cedex, France; \\ and ID17 Biomedical Beamline, European Synchrotron Radiation Facility (ESRF), 6 rue Jules Horowitz, \\ BP 220, 38043 Grenoble Cedex, France \\ C. Nemoz, M. Renier, and H. Requardt \\ ID17 Biomedical Beamline, European Synchrotron Radiation Facility (ESRF), 6 rue Jules Horowitz, \\ BP 220, 38043 Grenoble Cedex, France

\section{F. Estève} \\ Equipe 6, Institut National de la Santé et de la Recherche Médicale U836, 6 rue Jules Horowitz, BP 220, \\ 38043 Grenoble Cedex, France; Université Joseph Fourier, BP 53, 38041 Grenoble Cedex, France; \\ ID17 Biomedical Beamline, European Synchrotron Radiation Facility (ESRF), 6 rue Jules Horowitz, BP 220, \\ 38043 Grenoble Cedex, France; and Centre Hospitalier Universitaire, 38043 Grenoble, France \\ J. F. Adam \\ Equipe 6, Institut National de la Santé et de la Recherche Médicale U836, 6 rue Jules Horowitz, BP 220, \\ 38043 Grenoble Cedex, France; Université Joseph Fourier, BP 53, 38041 Grenoble Cedex, France; \\ and ID17 Biomedical Beamline, European Synchrotron Radiation Facility (ESRF), 6 rue Jules Horowitz, \\ BP 220, 38043 Grenoble Cedex, France
}

\section{H. Elleaume}

Equipe 6, Institut National de la Santé et de la Recherche Médicale U836, 6 rue Jules Horowitz, BP 220, 38043 Grenoble Cedex, France; Université Joseph Fourier, BP 53, 38041 Grenoble Cedex, France; ID17 Biomedical Beamline, European Synchrotron Radiation Facility (ESRF), 6 rue Jules Horowitz, BP 220, 38043 Grenoble, France; and Centre Hospitalier Universitaire, 38043 Grenoble, France

\section{A. Bravin \\ ID17 Biomedical Beamline, European Synchrotron Radiation Facility (ESRF), 6 rue Jules Horowitz, BP 220, 38043 Grenoble Cedex, France}

(Received 27 July 2008; revised 3 December 2008; accepted for publication 16 December 2008; published 4 February 2009)

Synchrotron radiation is an innovative tool for the treatment of brain tumors. In the stereotactic synchrotron radiation therapy (SSRT) technique a radiation dose enhancement specific to the tumor is obtained. The tumor is loaded with a high atomic number $(Z)$ element and it is irradiated in stereotactic conditions from several entrance angles. The aim of this work was to assess dosimetric properties of the SSRT for preparing clinical trials at the European Synchrotron Radiation Facility (ESRF). To estimate the possible risks, the doses received by the tumor and healthy tissues in the future clinical conditions have been calculated by using Monte Carlo simulations (PENELOPE code). The dose enhancement factors have been determined for different iodine concentrations in the tumor, several tumor positions, tumor sizes, and different beam sizes. A scheme for the dose escalation in the various phases of the clinical trials has been proposed. The biological equivalent doses and the normalized total doses received by the skull have been calculated in order to assure that the tolerance values are not reached. (C) 2009 American Association of Physicists in Medicine.

[DOI: $10.1118 / 1.3070538]$

Key words: clinical trials, synchrotron radiation, Monte Carlo simulations, biological equivalent dose, normalized total dose 


\section{INTRODUCTION}

Glioma is one of the most frequent primary brain tumors in adults, with an incidence of approximately 5-10 per 100000 general population. ${ }^{1}$ High-grade glioma is still of poor prognostic value despite the development of many innovative therapies. Stereotactic radiosurgery, ${ }^{2}$ intensity-modulated radiation therapy, ${ }^{3}$ and boron neutron capture therapy ${ }^{4}$ are some examples. Radiotherapy of such tumors requires high doses, whereas the tolerance of the healthy brain tissues limits the maximum allowable dose because of the high risk of normal tissue morbidity. ${ }^{5}$ The use of concomitant and adjuvant temozolomide and radiotherapy has allowed significant prolongation of survival. ${ }^{6,7}$ However, the outcome remains still unsatisfactory. Therefore the management of glioblastomas is still mainly palliative. The key parameter is to increase the radiation dose delivered to the tumor relative to the one absorbed by the healthy tissues. A radiation dose enhancement, specific to the tumor, can be achieved if the volume to be treated is previously loaded with an iodinated contrast agent and exposed to kilovoltage $\mathrm{x}$ rays.

This concept was first proposed by Norman and co-workers ${ }^{8-10}$ by using a simple irradiation scheme and a conventional scanner $\mathrm{x}$-ray source. The iodinated contrast media are injected to the patient and it accumulates preferentially in the tumor interstitium due to the increased permeability of the blood-brain barrier caused by invasive tumor growth. As already stressed by different authors, ${ }^{8,9,11}$ because of the local blood brain barrier disruption, tumor iodine concentrations of up to 4.0 and $5.0 \mathrm{mg} / \mathrm{ml}$ are routinely obtained by intravenous administration. These concentrations were observed using standard administration procedures for brain tumor imaging. With the specific aim of maximizing the iodine concentration, one may obtain tumor iodine concentrations of $10-20 \mathrm{mg} / \mathrm{ml}^{.}{ }^{8}$ Then, the iodine concentration achieved in the tumor undergoes a slow decay, whereas the iodine concentration in the surrounding normal tissue is negligible since no blood brain barrier leakage is observed in healthy brain tissue. At kilovoltage energies, the high photoelectric cross sections of iodine result in substantial photoelectric interactions [the atomic cross section for photoelectric absorption exhibits a $Z^{3}$ (Ref. 12)]. The high linear energy transfer and short range of the photoelectric interaction products (photoelectrons and Auger electrons) produce a localized dose enhancement. Therefore, the photon absorption is augmented specifically in the tumor volume and thus the absorbed dose is enhanced relatively to the surrounding tissues.

The dose enhancement yielded by this technique has been calculated previously by using Monte Carlo simulations. ${ }^{10,13}$ Its potential benefits and practical application have been demonstrated by the experimental treatment of tumor bearing rats, ${ }^{11,14}$ by the irradiation of human cells in vitro, ${ }^{9}$ and by the patient treatment of human brain metastasis by using a modified CT scanner without observing any bone toxicity or brain necrosis. ${ }^{10}$

The use of monochromatic $\mathrm{x}$ rays tuned at the optimal energy could significantly improve the dose distributions. ${ }^{15}$
Synchrotron sources, providing high-intensity, tunable monochromatic $\mathrm{x}$ rays, seem therefore ideal for this therapeutic modality. At the European Synchrotron Radiation Facility (ESRF), one beamline has been dedicated to medical applications of synchrotron radiation. ${ }^{16-19}$ At this beamline, SSRT preclinical trials were carried out in the past years in rats bearing high-grade F98 glioma. The survival of rats that received an intravenous injection of iodinated contrast agent before irradiation with monochromatic $50 \mathrm{keV} x$ rays was significantly prolonged. ${ }^{20,21}$

Based on those encouraging results, ESRF has decided to plan the clinical trials and the first patients are expected in the near future. With this objective, the doses received by the tumor and healthy tissues have to be assessed to define the irradiation protocol. In the clinical trials the tumor will be loaded with different concentrations of iodine, the beam size will be adjusted to the tumor dimensions, and the tumor positioned at the center of rotation. The irradiation will be performed over several incidences (maximum of 10) with a monochromatic beam of $80 \mathrm{keV}$. The choice of $80 \mathrm{keV}$ as the treatment energy among the technically possible ones at ESRF (from 20 to $100 \mathrm{keV}$ ) is based on the best compromise between the dose deposition in the tumor and the sparing of healthy tissues for a continuous arch irradiation. ${ }^{15}$ In previous works it has been shown that significant brain sparing is achieved in SSRT compared to conventional radiotherapy. In particular, comparisons have been performed with a stereotactic radiosurgery treatment (using $10 \mathrm{MV}$ beams) ${ }^{9}$ and with a conventional treatment with a $6 \mathrm{MV}$ linear accelerator. ${ }^{15}$ However, due to the high effective atomic number of bone (approximately 12.3) relative to tissue (approximately 7.5), particular care has to be applied to avoid complications to the bone. The tolerance dose for necrosis in the skull is $60 \mathrm{~Gy}$ in a standard fractionation scheme (2 Gy/session). ${ }^{22}$

In conventional radiotherapy, brain tumors are usually irradiated with $50 \mathrm{~Gy}$ in sessions of $2 \mathrm{~Gy}$. In the SSRT clinical trials, it is foreseen that the patients undergo first the SSRT treatment ("boost") and that 1 week later they start a total cerebral irradiation with conventional radiotherapy at the hospital to deliver additional $40 \mathrm{~Gy}$ to the tumor. The clinical trials will be carried out in different phases with an escalation in doses. The SSRT irradiation will be performed in one of the experimental stations of the Biomedical Beamline at the ESRF. During the days of the treatment, a complete priority will be given to the patient treatment and all other experimental activities in the beamline will be stopped. For that reason, single/hypofractionation schemes will be used. One of the goals of this work is to assess the possible dose prescriptions and fractionation scheme in order to optimize the number of sessions and the doses received by the skull in SSRT.

It is very important to take into account that the dose prescriptions will differ from the standard fractionation scheme of $2 \mathrm{~Gy} / \mathrm{session}$, and therefore the expected biological effects will be different. For that reason, the biological equivalent doses (BEDs) have been assessed. To establish the equivalence with the standard fractionation scheme and 
therefore to assess if the doses received by the skull are still within tolerances, the normalized total doses (NTDs) have been studied.

\section{MATERIALS AND METHODS}

\section{II.A. Monte Carlo source code}

To perform the dose calculations, a precise dosimetry system is required. However, none of the conventional treatment planning systems is able to compute dose distributions in the case of tumors loaded with high $Z$ elements and irradiated with kilovoltage $\mathrm{x}$ rays. Monte Carlo simulations are well suited for calculating dose distributions because cumulative doses are computed using track histories of each incident photon. Monte Carlo is therefore the most accurate and sensitive method available for calculating the dose enhancement with high $Z$ elements, as already shown in Refs. 9 and 15 .

In this work the PENELOPE code has been used. PENELOPE is a general purpose Monte Carlo simulation package developed at the University of Barcelona. ${ }^{23}$ The developers of this code put special emphasis on the implementation of accurate low energy electron cross sections, which are of particular importance for this application. The simulation algorithm is based on a scattering model that combines numerical databases with analytical cross section models for the different interaction mechanisms and is applicable to energies (kinetic energies in the case of electrons and positrons) from a few hundred $\mathrm{eV}$ to $1 \mathrm{GeV}$. It uses a mixed simulation scheme in which hard interactions are simulated collision by collision and small angular deflections and energy losses are treated in a grouped manner. That is to say, photon transport is simulated by means of the conventional detailed method. The simulation of electron and positron transport is performed by means of a mixed procedure. Hard interactions, with scattering angle or energy loss greater than preselected cutoff values, are simulated in detail. Soft interactions, with scattering angle or energy loss less than the corresponding cutoffs, are described by means of multiple scattering approaches. In this work, due to the fact that the beam energy is low $(80 \mathrm{keV})$ and there are high $Z$ elements present, the photoelectric interactions are of great relevance. PENELOPE incorporates a detailed description of photoabsorption in $K$ and $L$-shells (including the subsequent atomic relaxation). When the ionization occurs in an inner $K$ - or $L$-shell, the residual atom, with a vacancy in the shell, subsequently relaxes to its ground state by emitting $\mathrm{x}$ rays and Auger electrons. If the ionization occurs in an outer shell, it is assumed that the photoelectron leaves the target atom with kinetic energy equal to the energy deposited by the photon and the emission of subsidiary fluorescent radiation is disregarded. The photoelectric cross sections used in PENELOPE are obtained by interpolation in a numerical table that was extracted from the LLNL Evaluated Photon Data Library [EPDL (Ref. 24)]. The PENELOPE database for photoelectric absorption (a subset of the EPDL) consists of tables of the total atomic cross section $\theta_{\text {phi }}(E)$ and the cross sections for the $K$ - and $L$-shells, $\theta_{\text {phi, } i}(E)((i=K, L 1, L 2$ and $L 3)$ for the elements $Z=1-92$, which span the energy range from $100 \mathrm{eV}$ to $1000 \mathrm{GeV}$.

Compton scattering is as well a relevant photon interaction in low atomic number materials (like the brain) of interest in radiation therapy, for the $\mathrm{x}$-ray energies. ${ }^{25}$ PENELOPE considers bounding effects and Doppler broadening when simulating Compton interactions. The code has been widely used in medical-physics applications. ${ }^{26-30}$

In the simulations the heterogeneity is taken into account. The composition of a medium is specified by its stoichiometric formula, i.e., atomic number $Z_{i}$ and number of atoms per molecule $n_{i}$ of all the elements present. In this work, the material structure where radiation is transported consists of various regions with different compositions. In the code it is assumed that the interfaces between contiguous media are sharp (i.e., there is no diffusion of chemical species across them). When a particle arrives at an interface, it is stopped there and the simulation is resumed with the interaction properties of the new medium. This is based on the Markovian property of the transport process, that means that the future values of a random variable (interaction event) are statistically determined by present events and depend only on the event immediately preceding. Owing to the Markovian character of the transport, the generation of a particle history can be stopped at an arbitrary state (any point of the track) and the simulation can be resumed from this state without introducing any bias in the results.

In this work the 2006 version of the PENELOPE code was used. The number of primary photon stories is $10^{9}$ in all the calculations. The standard deviation is around $1 \%$. Some cross-checks have been performed with the code MNCPX (Ref. 31) and an average difference of $2 \%$ has been found between both codes. To be conservative, the $2 \%$ average difference has been taken as the error bar.

\section{II.B. Phantom description and simulated irradiation}

An anthropomorphic head phantom has been constructed with the geometry package in PENELOPE. The phantom is inspired by the one described in the work of Harling et al. ${ }^{32}$ for neutron capture therapy. It consists of three nonconcentric ellipsoids representing the skin, the skull, and the brain. A sagital and a coronal view of the phantom is represented in Fig. 1. In this model, the volume of the brain is $1470 \mathrm{~cm}^{3}$, those of the skull bone and of the skin are 1021 and $184 \mathrm{~cm}^{3}$, respectively.

A cylindrical tumor of $2 \mathrm{~cm}$ height and $2 \mathrm{~cm}$ of diameter was placed in different positions in the brain and loaded with different concentrations of iodine. Nine equiangular-spaced beams with an energy of $80 \mathrm{keV}$ and a size of $2 \times 2 \mathrm{~cm}^{2}$ irradiating the phantom have been simulated. An odd number of beams is used to avoid hot spots in the skull due to the possible overlap of opposite beams.

\section{II.C. Assessment of the dose enhancement factors}

The radiation dose enhancement is quantified by means of the dose enhancement factors (DEFs). The DEFs are defined as the ratio of the average radiation dose absorbed by the 


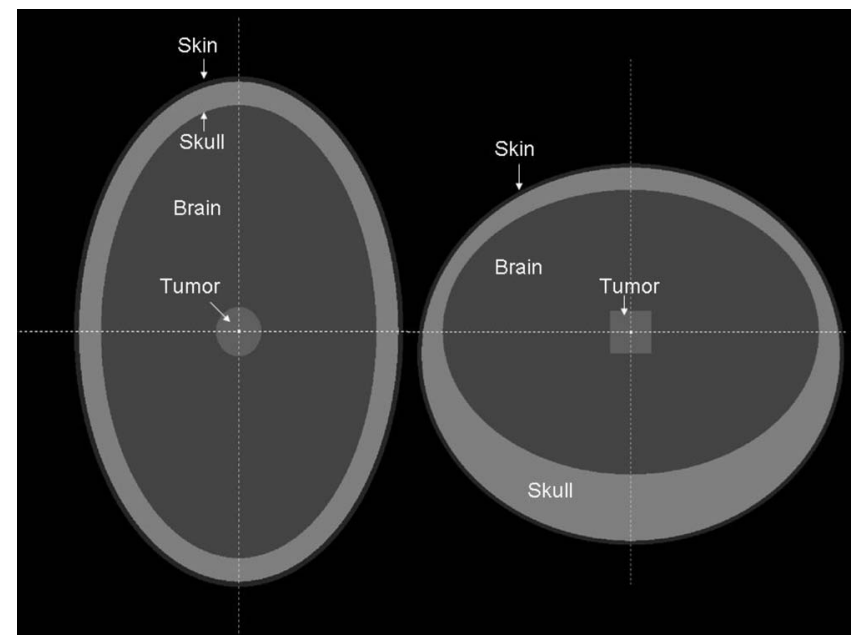

FIG. 1. Coronal (left) and sagital (right) views of the anthropomorphic phantom. Three nonconcentric ellipsoids simulate the skin, the skull, and the brain. A cylindrical tumor is placed at the center of the brain.

tumor when it is loaded with contrast media (iodine) to the dose absorbed without contrast media. The DEFs have been calculated by using Monte Carlo simulations.

\section{II.D. Assessment of the biological equivalent doses and normalized total doses}

To determine the value of the BED, expression (1) as derived by Fowler $^{33}$ from the linear-quadratic model has been used,

$$
\mathrm{BED}=n d\left(1+\frac{d}{(\alpha / \beta)}\right),
$$

where $n$ is the number of fractions, $d$ is the dose per fraction, and $\alpha$ and $\beta$ are parameter characteristics of the population of cells. To relate the biological effect of a course of radiation to the total dose of radiation administered with the standard fractionation scheme (2 Gy/fraction), the concept of normalized total dose (NTD) has been used. Expression (2) is derived from the linear-quadratic model, ${ }^{34}$

$$
\mathrm{NTD}=\mathrm{BED} /\left(1+\frac{d}{(\alpha / \beta)}\right) .
$$

To establish the equivalence with the standard fractionation scheme (2 Gy/fraction), in the Eq. (2), $d$ would take the value $2 \mathrm{~Gy}$. The $\alpha / \beta$ values are usually high for the tumors (except melanoma, liposarcoma, and prostate cancers ${ }^{35}$ ) and for early responding tissues (rapidily renewing tissues); the values are usually in the range between 10 and $15 \mathrm{~Gy}{ }^{35-37}$ For the late responding tissues, such as the bone, the $\alpha / \beta$ values are small, around $2 \mathrm{~Gy} .{ }^{35}$ Therefore, the effect of the dose fractionation and the repair between consecutive fractions is especially important for the late responding tissues, but it is less important for the tumor response and the early responding tissues, where the total dose is more relevant. There is a few published information about the $\alpha / \beta$ values of the bone. Overgaad ${ }^{38}$ estimated that the $\alpha / \beta$ ratios for the late bone damage are in the range from 1.8 to $2.8 \mathrm{~Gy}$. There-
TABLE I. Dose enhancement factors as a function of the iodine concentration in a cylindrical tumor of $2 \mathrm{~cm}$ of diameter and $2 \mathrm{~cm}$ height placed at the center of the brain.

\begin{tabular}{cc}
\hline \hline $\begin{array}{c}{[\mathrm{I}]} \\
(\mathrm{mg} / \mathrm{ml})\end{array}$ & DEF \\
\hline 1 & $1.10 \pm 0.01$ \\
5 & $1.47 \pm 0.01$ \\
8 & $1.74 \pm 0.02$ \\
9 & $1.84 \pm 0.02$ \\
10 & $1.93 \pm 0.02$ \\
15 & $2.37 \pm 0.02$ \\
20 & $2.80 \pm 0.03$ \\
\hline \hline
\end{tabular}

fore, to be conservative, the $\alpha / \beta$ value, which gives the highest BED in the bone, i.e., 1.8 Gy, has been used in the calculations. The value of $\alpha / \beta$ for the tumor was taken as 10 , which is an average of the values found for tumors in Ref. 39. This value has also been used in some other studies on gliomas. ${ }^{40,41}$

The linear-quadratic (LQ) model, used in this work, is a useful tool to assess the dose radiation response and to intercompare conventional fractionations since it closely fits the experimental cell survival curves in most of the cases of conventional fractionation schemes in radiotherapy. However, it is worth to notice that, although it is one of the best available models, it is not always compatible with clonogenic cell survival studies at high dose per fraction, where the survival curves do not really follow the continuous bending predicted by the LQ model. The LQ formulation overestimates the magnitude of cell kill for doses greater than $6 \mathrm{~Gy}$, and therefore, it underestimates the dose required to achieve a desired response for fractions of high dose. ${ }^{42}$ In addition it has also been shown that the linear-quadratic model is not so well suited to describe the response at low doses ( $\leq 1 \mathrm{~Gy})$ either. ${ }^{43}$

\section{RESULTS AND DISCUSSION}

This section presents the calculated DEF in a human head phantom for different iodine concentrations, different tumor positions, tumor sizes, and beam sizes, as well as the calculated doses received by the tumor and the skull.

\section{III.A. Dose enhancement factors as a function of the iodine concentration}

The variation in the DEF as a function of the iodine concentration in the tumor has been studied. In the simulations, a cylindrical tumor, as described in Sec. II B, has been used. Table I shows the DEF obtained for several iodine concentrations in the range between 0 and $20 \mathrm{mg} / \mathrm{ml}$. The variation in DEF versus iodine concentration follows a linear tendency $\left(r^{2}>0.999\right)$ in that range, in agreement with Ref. 44. 


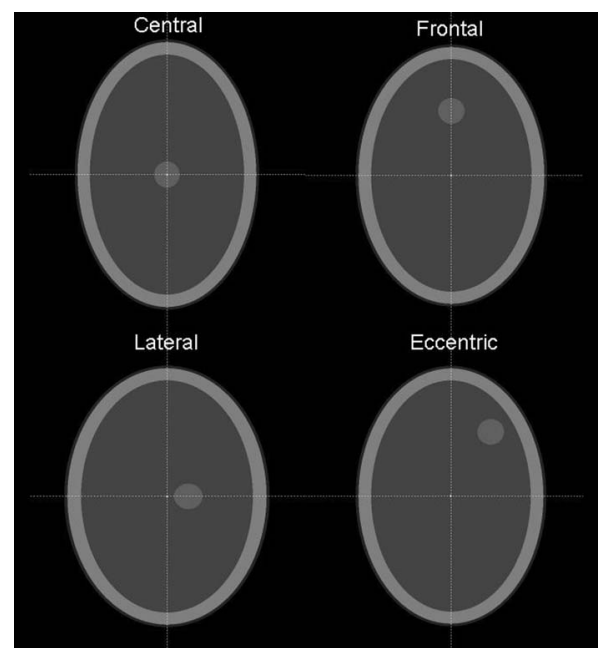

FIG. 2. Different tumor positions. Upper row left: Central. Upper row right: Frontal. Lower row left: Lateral. Lower row right: Eccentric.

\section{III.B. Dose enhancement factors as a function of the tumor position}

To study the variation in the DEF as a function of the tumor position, a cylindrical tumor (as defined in Sec. II B) loaded with $10 \mathrm{mg} / \mathrm{ml}$ of iodine was placed in four different positions: (1) Central position (from now on "central"), (2) displaced by $5 \mathrm{~cm}$ in the frontal direction ("frontal"), (3) displaced by $1.5 \mathrm{~cm}$ in the left-right direction ("lateral"), and (4) displaced by $3 \mathrm{~cm}$ in the left-right direction and by $4 \mathrm{~cm}$ in the frontal direction ("eccentric") (see Fig. 2). Table II shows that there is no variation in the DEF as a function of the tumor position within the error bars.

\section{III.C. Dose enhancement factors as a function of the tumor size}

The variation in the DEF as a function of the tumor size has also been investigated. One may expect a lower DEF with the increased tumor size due to strong attenuation in the tumor center. This would produce a depression at the center of the dose distribution in the tumor due to the high radiation absorption. This effect was found to be negligible for the concentrations expected to be used in the clinical trials, in particular, $10 \mathrm{mg} / \mathrm{ml}$. On the other hand, the higher the interaction volume, the higher will be the dose deposition in the tumor. DEF computations were carried out at increasing tumor diameters and heights with a constant $10 \mathrm{mg} / \mathrm{ml}$ iodine

TABLE II. Dose enhancement factors as a function of the tumor position. The tumor is loaded with an iodine concentration of $10 \mathrm{mg} / \mathrm{ml}$. No variation is observed within the error bars.

\begin{tabular}{lc}
\hline \hline Tumor position & DEF \\
\hline Central (center of the brain) & $1.93 \pm 0.02$ \\
Frontal ( $5 \mathrm{~cm}$ displaced in the frontal direction) & $1.92 \pm 0.02$ \\
Lateral (1.5 cm displaced in the lateral direction) & $1.91 \pm 0.02$ \\
Eccentric ( $3 \mathrm{~cm}$ displaced in the left-right direction and & \\
$4 \mathrm{~cm}$ in the frontal direction) & $1.92 \pm 0.02$ \\
\hline \hline
\end{tabular}

TABLE III. Dose enhancement factors as a function of the tumor size. The tumor is loaded with an iodine concentration of $10 \mathrm{mg} / \mathrm{ml}$. A slight increase in the DEF values with the tumor size is observed.

\begin{tabular}{cc}
\hline \hline $\begin{array}{c}\text { Tumor diameter/height } \\
(\mathrm{cm})\end{array}$ & DEF \\
\hline $0.5 / 0.5$ & $1.86 \pm 0.02$ \\
$1.0 / 1.0$ & $1.88 \pm 0.02$ \\
$1.5 / 1.5$ & $1.90 \pm 0.02$ \\
$2.0 / 2.0$ & $1.93 \pm 0.02$ \\
$2.5 / 2.5$ & $1.95 \pm 0.02$ \\
\hline \hline
\end{tabular}

concentration. A slight increase in the DEF with the tumor size was found and summarized in Table III. These results are not dependent on the tumor shape. Monte Carlo calculations of the DEF values in spherical tumors have proven that the significant parameter is the tumor volume.

\section{III.D. Dose enhancement factors as a function of the beam size}

To study the possible influence of the safety margins around the tumor, the beam sizes were varied in the Monte Carlo simulations. A cylindrical tumor as described in Sec. II B loaded with $10 \mathrm{mg} / \mathrm{ml}$ of iodine was placed at the center of the brain and irradiated with increasing beam sizes up to 1 $\mathrm{cm}$ of margin around the tumor $\left(3.0 \times 3.0 \mathrm{~cm}^{2}\right)$. Table IV shows that there is no variation in the DEF within the error bars up to the maximum margins expected in clinical conditions.

\section{III.E. Assessment of the doses received by the tumor and the skull}

As it has already been indicated in Sec. I, the SSRT treatments will be used as a boost to enhance the doses received by the tumor while sparing the surrounding tissues. Due to the high atomic number of the bone, the doses received by the skull can be important. The clinical trials will be carried out in different phases. One of the goals of this work is to study the possible dose prescriptions and fractionation scheme taking into account that the patient will receive an additional dose of $40 \mathrm{~Gy}$ in conventional radiotherapy. One possible scheme for the escalation of the prescribed doses to the tumor for the groups of patients to be treated in each of the phases of the clinical trials could be the following.

TABLE IV. Dose enhancement factors as a function of the beam size for a cylindrical tumor of $2 \mathrm{~cm}$ diameter and $2 \mathrm{~cm}$ height placed at the center of the brain and loaded with an iodine concentration of $10 \mathrm{mg} / \mathrm{ml}$. No variation is observed within the error bars.

\begin{tabular}{cc}
\hline \hline $\begin{array}{c}\text { Beam size } \\
\left(\mathrm{cm}^{2}\right)\end{array}$ & DEF \\
\hline $2.0 \times 2.0$ & $1.93 \pm 0.02$ \\
$2.2 \times 2.2$ & $1.93 \pm 0.02$ \\
$2.5 \times 2.5$ & $1.95 \pm 0.02$ \\
$3.0 \times 3.0$ & $1.97 \pm 0.02$ \\
\hline \hline
\end{tabular}


(1) First phase: $10 \mathrm{~Gy}$ in one session.

(2) Second phase: 12 Gy in one session.

(3) Third phase: 14 Gy in one session.

(4) Fourth phase: 18 Gy in three sessions.

(5) Fifth phase: $21 \mathrm{~Gy}$ in three sessions.

(6) Sixth phase: 24 Gy in four sessions.

To assess the doses received by the skull, the spatial dose distributions obtained from the Monte Carlo simulations have been studied and the maximum doses received by the skull in comparison with the average doses deposited in the tumor have been derived. Figure 3 shows the dose distributions at the central coronal plane of the geometry with a tumor placed at the center of the brain and loaded with different concentrations of iodine: 5,8 , and $10 \mathrm{mg} / \mathrm{ml}$. The maximum doses received by the skull were found to be $75 \%$, $69 \%$, and $65 \%$, respectively. That is to say, if the tumor receives $10 \mathrm{~Gy}$ in one session, then the maximum doses in the skull will be 7.5, 6.9, and $6.5 \mathrm{~Gy}$ in one session, respectively.

The proposed dose fractionation scheme differs from the standard one, and therefore the biological effects are expected to be different. To take this into account, the BEDs have been determined by using Eq. (1). To be able to assess the total doses received by the tumor and the skull, the $\mathrm{NTD}_{2.0}$ has been calculated by using Eq. (2). Tables V and VI show the maximum physical, BED, and $\mathrm{NTD}_{2.0}$ received by the tumor and the skull, respectively, in the proposed different phases of the clinical trials.

The total doses received by the tumor are calculated as the sum of the doses delivered in conventional radiotherapy (40 Gy) plus the calculated $\mathrm{NTD}_{2.0}$ received by the tumor in the SSRT treatment (see Table V). The total doses in the tumor, for the six phases proposed, are 57, 62, 68, 64, 70, and 72 Gy, respectively. This means an enhancement of the dose received by the tumor as compared with the one received in conventional radiotherapy (50 Gy). From the total cerebral irradiation with $40 \mathrm{~Gy}$ in conventional radiotherapy, a maximum dose of $20 \mathrm{~Gy}$ in the skull is expected. In addition, as it has already been explained in Sec. I, the tolerance dose for bone necrosis is $60 \mathrm{~Gy}$. Therefore, a $\mathrm{NTD}_{2.0}$ equal to $40 \mathrm{~Gy}$ can be considered a safe limit for the dose delivered to the skull in the SSRT treatment. As it can be seen in Table VI, the maximum $\mathrm{NTD}_{2.0}$ received by the skull is $30 \mathrm{~Gy}$ in the sixth phase and with an iodine concentration in the tumor of $5 \mathrm{mg} / \mathrm{ml}$. This maximum value still remains within tolerances.

In addition, the influence of the tumor position on the maximum doses received by the skull has been studied. The tumor was placed in the positions described in Sec. III B. The maximum doses received by the skull in comparison with the average doses deposited in the tumor have been determined for those tumor positions by using Monte Carlo simulations. Table VII shows the results when the tumor is loaded with a concentration of $10 \mathrm{mg} / \mathrm{ml}$ of iodine. The doses received by the skull are higher for the frontal and eccentric positions. Table VIII shows a comparison of the calculated maximum $\mathrm{NTD}_{2.0}$ received by the skull in the dif-
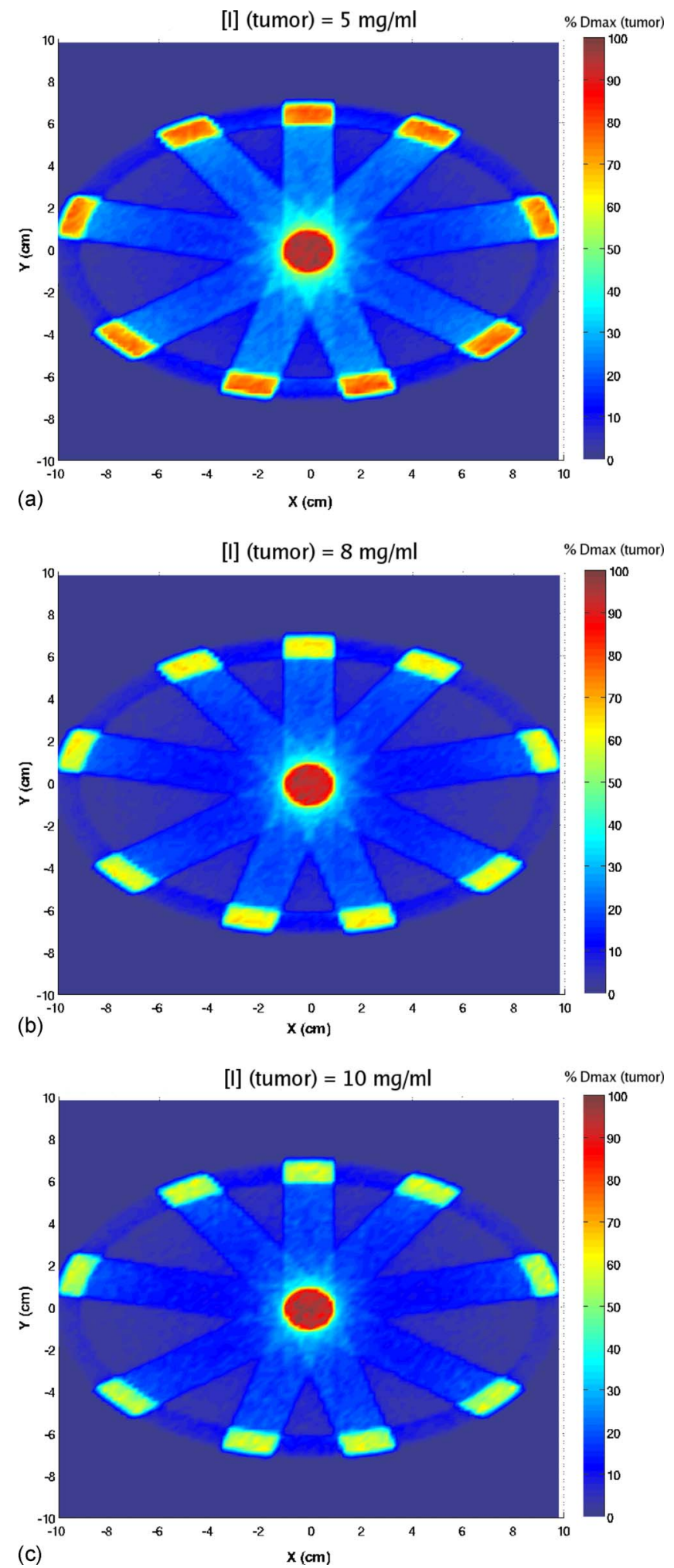

FIG. 3. Spatial dose distributions at the central coronal plane of the phantom for different iodine concentrations in the tumor. The doses are given in $\%$ of the maximum dose in the tumor. (a) Dose distribution for an iodine concentration of $5 \mathrm{mg} / \mathrm{ml}$. (b) Dose distribution for an iodine concentration of 8 $\mathrm{mg} / \mathrm{ml}$. (c) Dose distribution for an iodine concentration of $10 \mathrm{mg} / \mathrm{ml}$. 
TABLE V. Doses received by the tumor in the different phases of the clinical trials. The first column shows the dose prescription for the different phases, the second column is the physical dose in the tumor, and the third and fourth columns show the normalized total doses and the biological equivalent doses received by the tumor, respectively. The doses have been rounded to integer values.

\begin{tabular}{cccc}
\hline \hline Dose prescription & $\begin{array}{c}D \text { (tumor) } \\
(\mathrm{Gy})\end{array}$ & $\begin{array}{c}\mathrm{NTD}_{2.0} \text { (tumor) } \\
(\mathrm{Gy})\end{array}$ & $\begin{array}{c}\mathrm{BED} \text { (tumor) } \\
\left(\mathrm{Gy}_{10}\right)\end{array}$ \\
\hline $10 \mathrm{~Gy} / 1 \mathrm{frac}$. & 10 & 17 & 20 \\
$12 \mathrm{~Gy} / 1 \mathrm{frac}$. & 12 & 22 & 26 \\
$14 \mathrm{~Gy} / 1 \mathrm{frac}$. & 14 & 28 & 34 \\
$18 \mathrm{~Gy} / 3 \mathrm{frac}$. & 18 & 24 & 29 \\
$21 \mathrm{~Gy} / 3$ frac. & 21 & 30 & 36 \\
$24 \mathrm{~Gy} / 4 \mathrm{frac}$. & 24 & 32 & 38 \\
\hline \hline
\end{tabular}

ferent phases for the four tumor positions. The tumor is loaded with an iodine concentration of $10 \mathrm{mg} / \mathrm{ml}$. As it can be seen, all the doses remain below $40 \mathrm{~Gy}$, and therefore within the tolerance level.

TABLE VI. Assessment of the maximum doses received by the skull in the different phases of the clinical trials. The first column shows different iodine concentrations, the second and third columns show the maximum physical dose, the normalized total doses and the biological equivalent doses received by the skull when the tumor is loaded with those iodine concentrations, respectively. The doses have been rounded to integer values.

\begin{tabular}{|c|c|c|c|}
\hline $\begin{array}{c}{[\mathrm{I}]} \\
(\mathrm{mg} / \mathrm{ml})\end{array}$ & $\begin{array}{c}D_{\max }(\text { skull }) \\
\text { (Gy) }\end{array}$ & $\begin{array}{c}\mathrm{NTD}_{2.0, \max }(\text { skull) } \\
\text { (Gy) }\end{array}$ & $\begin{array}{c}\mathrm{BED}_{\max } \text { (skull) } \\
\left(\mathrm{Gy}_{1.8}\right)\end{array}$ \\
\hline Phase 1: & $10 \mathrm{~Gy} / 1 \mathrm{frac}$. & & \\
\hline 5 & $7.5 \pm 0.1$ & 18 & 39 \\
\hline 8 & $6.9 \pm 0.1$ & 16 & 33 \\
\hline 10 & $6.5 \pm 0.1$ & 14 & 30 \\
\hline Phase 2: & $12 \mathrm{~Gy} / 1$ frac. & & \\
\hline 5 & $9.0 \pm 0.1$ & 26 & 54 \\
\hline 8 & $8.3 \pm 0.1$ & 22 & 46 \\
\hline 10 & $7.8 \pm 0.1$ & 20 & 42 \\
\hline Phase 3: & 14 Gy/1 frac. & & \\
\hline 5 & $10.5 \pm 0.1$ & 34 & 72 \\
\hline 8 & $9.7 \pm 0.1$ & 29 & 62 \\
\hline 10 & $9.1 \pm 0.1$ & 26 & 55 \\
\hline Phase 4: & $18 \mathrm{~Gy} / 3 \mathrm{frac}$. & & \\
\hline 5 & $13.5 \pm 0.1$ & 22 & 47 \\
\hline 8 & $12.4 \pm 0.1$ & 19 & 41 \\
\hline 10 & $11.7 \pm 0.1$ & 18 & 37 \\
\hline Phase 5: & $21 \mathrm{~Gy} / 3$ frac. & & \\
\hline 5 & $15.8 \pm 0.1$ & 29 & 62 \\
\hline 8 & $14.5 \pm 0.1$ & 25 & 53 \\
\hline 10 & $13.6 \pm 0.1$ & 23 & 48 \\
\hline Phase 6: & 24 Gy/4 frac. & & \\
\hline 5 & $18.0 \pm 0.1$ & 30 & 63 \\
\hline 8 & $16.6 \pm 0.1$ & 26 & 55 \\
\hline 10 & $15.6 \pm 0.1$ & 23 & 49 \\
\hline
\end{tabular}

TABLE VII. Ratio of the maximum doses received by the skull and the average doses in the tumor for the different tumor positions.

\begin{tabular}{lc}
\hline \hline Tumor position & $\begin{array}{c}\text { Dose }_{\max } \text { skull/ } / D_{\mathrm{av}} \text { tumor } \\
(\%)\end{array}$ \\
\hline Central & 65 \\
Frontal & 73 \\
Lateral & 65 \\
Eccentric & 71 \\
\hline \hline
\end{tabular}

The calculations show that in SSRT it is possible to enhance the dose delivered to the tumor while keeping the doses in the skull within the tolerance level. The Monte Carlo simulations show that the DEF increases linearly with the iodine concentration. The measurement of the iodine concentration in the tumor must be accurate because small differences in the iodine concentration lead to relevant differences in doses. The DEF values do not show any dependence on the tumor position. However an increase in the tumor size is observed ( $<5 \%$ for the range of tumor sizes to be treated in the clinical trials). In addition, to study the effects of the safety margins around the tumor, the irradiation beam sizes were increased up to the maximum margins expected $(1 \mathrm{~cm})$ and no variation in DEF was observed.

The BED and NTD studies have shown that it is possible to obtain an enhancement in the dose received by the tumor compared with the one received in conventional radiotherapy (50 Gy). This enhancement can be as high as 22 Gy in the sixth phase. This might increase the probability of tumor control. On the other hand, in spite of the high atomic number of the bone, it has been shown that the $\mathrm{NTD}_{2.0}$ in the skull remain within the acceptable level for the dose escalation proposed and the irradiation geometry foreseen at ESRF for the SSRT clinical trials.

\section{CONCLUSIONS}

The stereotactic synchrotron radiation therapy is a promising technique to treat brain tumors. The goal is to enhance the dose delivered to the tumor by loading the tumoral tissue with iodine and irradiating it with monochromatic $\mathrm{x}$ rays. In this work the doses received by the tumor and the skull in the future conditions of the clinical trials have been assessed by using Monte Carlo simulations. The DEF values have been

TABLE VIII. NTD 2.0 received by the skull for the four different positions of the tumor (iodine concentration of $10 \mathrm{mg} / \mathrm{ml}$ ) and in the different phases of the clinical trials. All the doses are in Gy.

\begin{tabular}{ccccc}
\hline \hline Dose prescription & $\begin{array}{c}\mathrm{NTD}_{2.0} \\
(\text { Central })\end{array}$ & $\begin{array}{c}\mathrm{NTD}_{2.0} \\
\text { (Frontal) }\end{array}$ & $\begin{array}{c}\mathrm{NTD}_{2.0} \\
\text { (Lateral) }\end{array}$ & $\begin{array}{c}\mathrm{NTD}_{2.0} \\
(\text { Eccentric })\end{array}$ \\
\hline $10 \mathrm{~Gy} / 1$ frac. & 14 & 18 & 14 & 17 \\
$12 \mathrm{~Gy} / 1$ frac. & 20 & 24 & 20 & 23 \\
$14 \mathrm{~Gy} / 1 \mathrm{frac}$ & 26 & 32 & 26 & 31 \\
$18 \mathrm{~Gy} / 3$ frac. & 18 & 21 & 18 & 20 \\
$21 \mathrm{~Gy} / 3$ frac. & 23 & 28 & 23 & 27 \\
$24 \mathrm{~Gy} / 4$ frac. & 23 & 28 & 23 & 27 \\
\hline \hline
\end{tabular}


studied as a function of the iodine concentration in the tumor, of the tumor position, tumor size, and beam sizes. A scheme for the dose escalation in the different phases of the clinical trials has been proposed. The BEDs received by the tumor and the skull have been calculated in order to establish an equivalence with the standard fractionation scheme of $2 \mathrm{~Gy} /$ session. It has been shown that an enhancement in the doses received by the tumor compared to conventional radiotherapy can be obtained in SSRT. Therefore, an increase in the tumor control probability is expected, whereas the doses received by the skull remain within the tolerance level. This work reflects as well the importance of the inclusion of the biological aspects in the dose calculations.

a) Electronic addresses: prezado@esrf.fr

${ }^{1}$ J. M. Legler et al., "Cancer surveillance series [corrected]: Brain and other central nervous system cancers: Recent trends in incidence and mortality," J. Natl. Cancer Inst. 91, 1382-1390 (1999).

${ }^{2}$ M. H. Phillips et al., "Stereotactic radiosurgery: A review and comparison of methods," J. Clin. Oncol. 12, 1085-1099 (1994).

${ }^{3}$ R. M. Cardinale et al., "Comparison of three stereotactic radiotherapy techniques: ARCS vs. noncoplanar fixed fields vs. intensity modulation," Int. J. Radiat. Oncol., Biol., Phys. 42, 431-436 (1998).

${ }^{4}$ R. F. Barth et al., "Boron neutron capture therapy of cancer: Current status and future prospects," Clin. Cancer Res. 11, 3987-4002 (2005).

${ }^{5}$ A. R. Kagan et al., "The pathogenesis of brain necrosis: Time and dose parameters,” Int. J. Radiat. Oncol., Biol., Phys. 1, 729-732 (1977).

${ }^{6} \mathrm{R}$. Stupp et al., "Radiotherapy plus concomitant and adjuvant temozolomide for glioblastoma," N. Engl. J. Med. 352, 987-996 (2005).

${ }^{7}$ R. Stupp et al., "Promising survival for patients with newly diagnosed glioblastoma multiforme treated with concomitant radiation plus temozolomide followed by adjuvant temozolomide," J. Clin. Oncol. 20, 13751382 (2002).

${ }^{8}$ A. Norman, K. S. Iwamoto, and S. T. Cochran, "Iodinated contrast agents for brain tumor localization and radiation dose enhancement," Invest. Radiol. 26, 120-121 (1991).

${ }^{9}$ A. V. Mesa, A. Norman, T. D. Solberg, J. J. Demarco, and J. B. Smathers, "Dose distributions using kilovoltage $\mathrm{x}$-rays and dose enhancement from iodine contrast agents," Phys. Med. Biol. 44, 1955-1968 (1999).

${ }^{10}$ J. H. Rose, A. Norman, M. Ingram, C. Aoki, T. Solberg, and A. Mesa, "First radiotherapy of human metastatic brain tumors delivered by a computerized tomography scanner (CTRx)," Int. J. Radiat. Oncol., Biol., Phys. 45, 1127-1132 (1999).

${ }^{11}$ R. S. Mello, H. Callisen, J. Winter, A. Robert Kagan, and A. Norman, "Radiation dose enhacement in tumors with iodine," Med. Phys. 10, 75-78 (1983).

${ }^{12}$ F. M. Khan, The Physics of Radiation Therapy, 3rd ed. (Lippincott Williams and Wilkins, Philadelpha, 2003).

${ }^{13}$ T. D. Solberg, K. S. Iwamoto, and A. Norman, "Calculation of radiation dose enhancement factors for dose enhancement therapy of brain tumors," Phys. Med. Biol. 37, 439-443 (1992).

${ }^{14} \mathrm{~K}$. S. Iwamoto et al., "Radiation dose enhancement therapy with iodine in rabbit VX-2 brain tumors," Radiother. Oncol. 8, 161-170 (1987).

${ }^{15}$ C. Boudou, J. Balosso, F. Estève, and H. Elleaume, "Monte Carlo dosimetry for stereotactic synchrotron radiation therapy of brain tumors," Phys. Med. Biol. 50, 4841-4851 (2005).

${ }^{16} \mathrm{P}$. Suortti and W. Thomlinson, "Medical applications of synchrotron radiation," Phys. Med. Biol. 48, R1-R35 (2003).

${ }^{17} \mathrm{~B}$. Bertrand et al., "Comparison of synchrotron radiation angiopgraphy with conventional angiography for the diagnosis of in-stent restenosis after percutaneous transluminal coronary angiography," Eur. Heart J. 26, 1284-1291 (2005).

${ }^{18}$ R. Serduc et al., "Characterization and quantification of cerebral edema induced by synchrotron x-ray microbeam radiation therapy," Phys. Med. Biol. 53, 1153-1166 (2008).

${ }^{19} \mathrm{~A}$. Bravin, "The biomedical programs at the ID17 beamline of the European synchrotron Radiation Facility," in Brilliant Light in Life and Materials Sciences, NATO Advanced Studies Institute, Series B: Physics and Biophysics (Springer, The Netherlands, 2007), pp. 225-239.
${ }^{20}$ J. F. Adam et al., "Synchrotron radiation therapy of malignant brain glioma loaded with an iodinated contrast agent: First trial on rats bearing F98 gliomas," Int. J. Radiat. Oncol., Biol., Phys. 57, 1413-1426 (2003).

${ }^{21}$ J. F. Adam et al., "Prolonged survival of Fischer rats bearing F98 glioma after iodine-enhanced synchrotron stereotactic radiotherapy," Int. J. Radiat. Oncol., Biol., Phys. 64, 603-611 (2006).

${ }^{22}$ B. Emami et al., "Tolerance of normal tissue to therapeutic irradiation," Int. J. Radiat. Oncol., Biol., Phys. 21, 109-122 (1991).

${ }^{23}$ F. Salvat, J. M. Fernández-Varea, and J. Sempau, PENELope, a code system for Monte Carlo simulation of electron and photon transport, OECD Nuclear Energy Agency, Issy-les-Moulineaux-France, 2003 (available in PDF format from the web at www.nea.fr).

${ }^{24}$ D. E. Cullen, J. H. Hubbell, and L. Kissel, "EPDL97 The evaluated data library, 097 version," Lawrence Livermore National Laboratory Report No. UCRL-50400, 1997.

${ }^{25}$ J. Stepanek, H. Blattman, J. A. Laissue, N. Lyubimova, M. De Michiel, and D. N. Slatkin, "Physics study of microbeam radiation therapy with PSI version of Monte Carlo code GEANT as a new computational tool," Med. Phys. 27, 1664-1675 (2000).

${ }^{26}$ J. Sempau, A. Sanchez-Reyes, F. Salvat, H. O. Ben Tahar, S. B. Jiang, and J. M. Fernández-Varea, "Monte Carlo simulation of electron beams from an accelerator head using PENELope," Phys. Med. Biol. 46, 11631186 (2001).

${ }^{27}$ A. Badano and J. Sempau, "MANTIS: Combined x-ray, electron and optical Monte Carlo simulations of indirect radiation imaging systems," Med. Phys. 33, 2698-2713 (2006).

${ }^{28}$ J. Asenjo, J. M. Fernández-Varea, and A. Sanchez-Reyes, "Characterization of a high-dose-rate Sr-90-Y-90 source for intravascular brachytherapy by using the Monte Carlo code PENElope," Phys. Med. Biol. 47, 697-711 (2002).

${ }^{29}$ V. Moskvin, R. Timmerman, C. DesRosiers, M. Randall, P. Des Rosiers, P. Dittmer, and L. Papiez, "Monte Carlo simulation of the Leksell gamma Knife (R). II. Effects of heterogeneous versus homogeneous media for stereotactic radiosurgery," Phys. Med. Biol. 49, 4879-4895 (2004).

${ }^{30}$ E. Siegbahn, J. Stepanek, E. Brauer-Krisch, and A. Bravin, "Determination of dosimetrical quantities used in microbeam radiation therapy (MRT) with Monte Carlo simulations," Med. Phys. 33, 3248-3259 (2006).

${ }^{31}$ J. S. Hendricks et al., MCNPX, Version 2.5.e, Los Alamos Laboratory, Los Alamos, NM, 2004.

${ }^{32}$ O. K. Harling, K. A. Roberts, D. J. Moulin, and R. D. Rogus, "Head phantoms for neutron capture therapy," Med. Phys. 22, 579-583 (1995).

${ }^{33}$ J. F. Fowler, "The linear-quadratic formula and progress in fractionated radiotherapy," Br. J. Radiol. 62, 679-694 (1989).

${ }^{34}$ J. C. Flickinger and A. Kalend, "Use of normaliyed total dose to represent the biological effect of fractionated radiotherapy," Radiother. Oncol. 17, 339-347 (1990).

${ }^{35}$ D. J. Brenner et al., "Direct evidence that prostate cancers show high sensitivity to fractionation, similar to late-responding normal tissue," Int. J. Radiat. Oncol., Biol., Phys. 52, 6-13 (2002).

${ }^{36}$ S. M. Bentzen, A. C. C. Ruifrok, and H. D. Thames, "Repair capacity and kinetics for human mucosa and epithelial tumors in the head and neck: Clinical data on the effect of changing the time interval between multiple fractions per day in radiotherapy," Radiat. Oncol. 38, 89-101 (1996).

${ }^{37}$ M. V. Willians, J. Denekamp, and J. F. Fowler, "A review of alfa/beta ratios for experimental tumors: Implications for clinical studies of altered fractionation,” Int. J. Radiat. Oncol., Biol., Phys. 11, 87-96 (1985).

${ }^{38} \mathrm{M}$. Overgaard, "Spontaneous radiation-induced rib fractures in breast cancer patients treated with postmastectomy irradiation: A clinical radiobiological analysis of the influence of fraction size and dose-response relationships on late bone damage," Acta Oncol. 27, 117-22 (1988).

${ }^{39}$ G. G. Steel, G. E. Adams, and A. Horwich, The Biological Basis of Radiotherapy, 2nd ed. (Elsevier, New York, 1989).

${ }^{40}$ Y. Shibamoto et al., "Comparison of accelerated hyperfractionation radiotherapy and conventional radiotherapy for supratentorial malignat glioma,” Jpn. J. Clin. Oncol. 27, 31-36 (1997).

${ }^{41}$ J. L. Chan et al., "Survival and failure patterns of high-grade gliomas after three-dimensional conformal radiotherapy," Int. J. Clin. Oncol. 20, 1635-1642 (2002). 
${ }^{42}$ K. G. Zimmer, Studies on Quantitative Radiation biology (Oliver and Boyd, Edinburgh, 1961).

${ }^{43}$ P. Lambin, E. P. Malaise, and M. C. Joiner, "The effect of very low radiation doses on the human bladder carcinoma cell line RT112," Radio- ther. Oncol. 32, 63-72 (1994).

${ }^{44}$ K. N. Morris, M. D. Weil, and R. Malzbender, "Radiochromic film dosimetry of contrast enhanced RT (CERT)," Phys. Med. Biol. 51, 59155925 (2006). 